\title{
Features of the deformation of glass- and carbon-reinforced plastics based on T-107 binder under tension
}

\author{
Yu. E. Kalinin 1 , O. A. Karaeva ${ }^{2}$, A. T. Kosilov ${ }^{1}$, A. M. Kudrin ${ }^{\dagger, 2}$, O. V. Ovdak ${ }^{1}$ \\ †kudrin.ru@gmail.com \\ ${ }^{1}$ Voronezh State Technical University, 14 Moskovskii Av., Voronezh, 394026, Russia \\ ${ }^{2} J S C$ “Rusaviainter”, 31-V Leningradskaya St., Voronezh, 394004, Russia
}

\begin{abstract}
The article presents the results of a study on the mechanical properties of glass (T-10-14 filler) and carbon-fiber (Formosa TC-35 12K) reinforced plastics in an epoxy matrix T-107. It was found that the mechanical properties of carbon fiber were superior to fiberglass. Regardless of the composition of the composite, there are two distinct stages on the deformation curves of experimental samples based both on carbon and glass fibers, which correspond to elastic deformation and softening stages associated with the failure of composite fibers. Taking account for the significant difference between the elastic moduli and tensile strength of carbon and glass fibers, on the one hand, and the polymer matrix, on the other, it was suggested that the observed effect of softening of the composites is associated with the process of sequential breaking of the fibers as the applied load increases. Basing on the proposed idea, a model of deformation of a polymer composite material based on the sequential fracture of the filler fiber with increasing the applied load has been developed. According to this model, the observed increase in the applied load at the stage of strain hardening is associated with the presence of dispersion of the fibers of the samples by the values of strength, i. e. there is a distribution of the strength characteristics of the fiber along the length. With an increase in the length of a fiber in the sample, and, consequently, the length of the sample itself, the probability of appearance of thread sections with an even lower level of strength increases. Thus, in general, the tensile strength of the composite measured in the experiment should decrease. This dependence of the strength characteristics of the composite on the geometrical parameters of the product is determined by the fiber strength distribution function along its length, which can be determined from the experimental curves of tensile specimens of composites. Taking into account the proposed model, the obtained experimental results are used to estimate the distribution function $g(f)$ of the probability of detection of its strength value per unit length of fiber.
\end{abstract}

Keywords: CFRP, fiberglass, tensile strength.

УДК: $539.4,678.046$

\section{Особенности деформации стекло- и углепластиков с матрицей Т-107 при растяжении}

\author{
Калинин Ю. Е. ${ }^{1}$, Караева О. А. ${ }^{2}$, Косилов А. Т. ${ }^{1}$, Кудрин А. М. ${ }^{\dagger}$, , Овдак О. В. ${ }^{1}$ \\ ${ }^{1}$ Воронежский государственный технический университет, Московский пр., 14, Воронеж, 394026, Россия \\ ${ }^{2} \mathrm{AO}$ «Русавиаинтер», ул. Ленинградская, 31-В, 394004, Воронеж, Россия
}

В статье представлены результаты исследования механических свойств стекло- и углепластиков с наполнителем T-10-14 и Formosa TC-35 12K, соответственно, в эпоксидной матрице Т-107. Установлено, что углепластики по механическим свойствам превосходят стеклопластики. Независимо от состава композита, на деформационных кривых экспериментальных образцов, как на основе углеродных волокон, так и стеклянных волокон, наблюдаются две ярко выраженные стадии, соответствующие упругой деформации и стадии разупрочнения, связанной с разрушением волокон композита. Принимая во внимание существенную величину разницы между модулями упругости и пределом прочности углеродных и стеклянных волокон, с одной стороны, и полимерной матрицы с другой, в работе предположено, что наблюдаемый эффект разупрочнения композитов связан с процессом последовательного разрыва волокон по мере роста приложенной нагрузки. С учетом предложенной идеи разра- 
ботана модель деформации полимерного композиционного материала, основанная на последовательном разрыве волокон наполнителя по мере роста приложенной нагрузки. Согласно этой модели, наблюдаемый рост приложенной нагрузки на стадии деформационного упрочнения связан с наличием у волокон образцов разброса по уровню прочности, то есть имеет место распределение прочностных характеристик волокна по длине. С ростом длины волокна в образце, а, следовательно, длины самого образца, растет вероятность появления участков нити с все более низким уровнем прочности. При этом, в общем случае, должен понижаться измеряемый в эксперименте предел прочности композита. Эта зависимость прочностных характеристик композита от геометрических параметров изделия определяется функцией распределения прочности волокна по его длине, которая может быть определена по экспериментальным деформационным кривым растяжения образцов композитов. С учетом предложенной модели по полученным экспериментальным результатам сделана оценка функции распределения $g(f)$ вероятности обнаружения на единице длины волокна величины его прочности.

Ключевые слова: углепластик, стекловолокно, предел прочности.

\section{1. Введение}

Полимерный композит представляет собой материал, изготовленный путем объединения двух и более разнородных фаз (компонентов) с образованием нового материала с принципиально иными свойствами, которые существенно отличаются от свойств его компонентов по отдельности. Армирование полимерной матрицы стеклянными и/или углеродными волокнами привело к открытию нового класса материалов, удовлетворяющего жестким требованиям, предъявляемым оборонной, авиационной и космической промышленностью - сочетание высоких прочностных характеристик с низким удельным весом [1-2]. Особое место среди высокопрочных полимерных композиционных материалов (ПКМ) занимают стеклоуглепластики или гибридные ПКМ, для которых при малой плотности характерно сочетание высокой жесткости, прочности, термостойкости, высоких значений межфазной связи, предопределяющих стойкость к ударным нагрузкам [3]. Изделия из ПКМ на основе углеродного наполнителя проявляют достаточно низкое сопротивление кратковременным механическим воздействиям. Внимание ученых направлено на повышение эффективности воздействия композитов ударным и растягивающим нагрузкам при низких скоростях нагружения (нагрузки малых скоростей - до 10 м/с). Среди основных методов выделяются специальная обработка волокон, определенные схемы их чередования, матричная модификация [4-5]. Существенно повысить вязкость разрушения можно с использованием гибридных ПКМ - стеклоуглепластиков. Гибридизация представляет собой комбинацию двух и более видов армирующих наполнителей и часто обусловлена не только необходимостью сопротивления вязкостному разрушению, но и снижению стоимости ПКМ за счет сочетания дорогостоящих материалов с более дешевыми (стеклянными). Одновременное применение в композите стеклянных и углеродных волокон позволяет с одной стороны достичь высокой межфазной прочности, достаточной для переноса нагрузки с полимерной матрицы на волокна, с другой - проявить демпфирующий эффект, препятствующий расслоению материала при нагрузках различной природы [6].
Важной эксплуатационной характеристикой, определяющей применение волокнистых ПКМ в различных конструкциях, является их сопротивление растягивающим нагрузкам, которые зависят от прочности и модуля упругости волокон и матрицы. При этом разрушение композиционных материалов, армированных непрерывными однонаправленными волокнами, является многостадийным многофакторным процессом, охватывающим различные масштабные и структурные уровни материала [7].

Для описания прочности при растяжении однонаправленного композиционного материала обычно применяют модели, основанные на правиле смеси или аддитивности [8]. В идеальных условиях предполагается, что все фазы при разных уровнях нагружения находятся в условиях равной деформации, волокна расположены параллельно оси нагружения, связь между волокнами и матрицей является совершенной, свойства фаз остаются теми же, что и до объединения в композиционный материал, взаимодействие волокна с матрицей не приводит к появлению поперечных напряжений, при этом до нагружения в составляющих композиционного материала нет остаточных напряжений [9]. В реальных условиях механических испытаний возникает ряд отклонений от идеальных условий, используемых в моделях, что усложняет процесс разрушения ПКМ.

Следовательно, изучение особенностей упругопластичного поведения и разрушения ПКМ, армированного непрерывными волокнами является актуальной задачей, определяющей эксплуатационные качества композиционного материала и элементов конструкции, изготовленной из ПКМ. Для оценки эксплуатационных свойств композиционного материала необходимо применять прямые и теоретические методы исследований, которые в совокупности могут дать полное представление о прочностных, упругих параметрах материала и механизме разрушения. C учетом вышесказанного целью предлагаемой работы было исследование особенностей разрушения при разрыве ПКМ на основе угле- и стекловолокон в матрице расплавного эпоксидного связующего марки Т-107, состав которого разработан химическим факультетом МГУ им. М.В. Ломоносова [10]. 


\section{2. Методики получения и исследования}

Методики получения образцов и исследования механических свойств описаны в $[11,12]$. Образцы ПКМ для испытания на растяжение представляли собой полосы прямоугольного сечения с закрепленными на концах накладками из ортогонально направленного пластика КАСТ. Геометрические размеры образцов на растяжение составляли $250 \times 15 \times 1 \mathrm{mм}^{3}$. Количество образцов ПКМ, необходимое для определения предела прочности при растяжении и предельной деформации в заданном направлении композиционного материала одной партии, составляло не менее пяти [13]. Образцы ПКМ на основе углеродных (УВ) и стеклянных волокон (СВ) имели идентичные геометрические размеры. Образцы монолитного связующего для определения модуля упругости эпоксидной матрицы Т-107 изготавливали в виде лопатки с геометрическими размерами, представленными в [14].

Испытания проводили на разрывной универсальной машине Instron 5985 с максимальным усилием 250 кН c применением гидравлической станции Instron А569-101, обеспечивающей растяжение образца с постоянной скоростью перемещения траверсы $V=2$ мм/мин, в строгом соответствии с требованиями методик стандартов ASTM D 3039 [13] и ASTM D 638 [14]. Температура испытания составляла $t=20^{\circ} \mathrm{C}$.

Для измерения деформации и модуля упругости использовали экстензометр 2630-111 (база 50 мм), который устанавливался непосредственно на образец.

Напряжение при растяжении рассчитывали по формуле:

$$
\sigma=P / S,
$$

где $\sigma-$ напряжение при растяжении, МПа; $P-$ прикладываемая нагрузка, $\mathrm{H} ; S-$ средняя площадь поперечного сечения, мм².

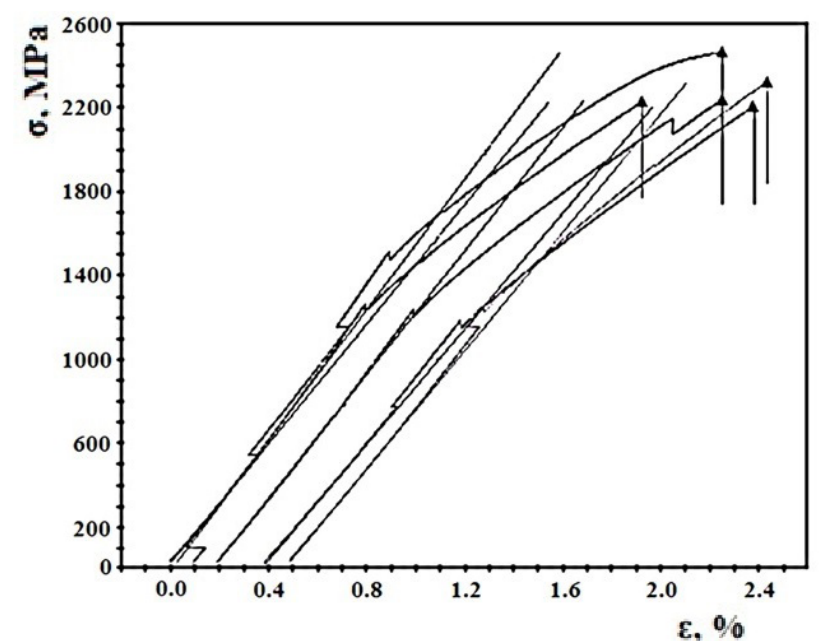

a
Относительное удлинение при разрыве $\varepsilon, \%$ определяли по формуле:

$$
\varepsilon=\left[\left(l_{k}-l_{0}\right) / l_{0}\right] \cdot 100 \%,
$$

где $l_{k}$ - длина образца после разрыва, мм; $l_{0}$ - длина образца до испытания, мм.

\section{3. Результаты эксперимента и их анализ}

Результаты испытаний на растяжение синтезированных образцов композиционных материалов с различным наполнителем представлены на Рис. 1. Наиболее высокие значения прочностных свойств наблюдаются у композитов с наполнителем из углепластика Formosa ТС-35-12К (Рис. 1a), а наиболее низкие - с наполнителем из стеклопластика Т-10-14 (Рис. 1b).

Результаты испытаний на растяжение монолитного связующего для определения модуля упругости эпоксидной матрицы Т-107 представлены на Рис. 2.

Анализ полученных результатов показал, что независимо от состава композита - на деформационных кривых композитов, как на основе УВ, так и СВ, наблюдаются, как это показано на Рис. 2, две ярко выраженных стадии: упругой деформации и стадии разупрочнения, связанной с разрушением волокнистого композита. Принимая во внимание существенную величину разницы между модулями упругости и пределом прочности УВ и СВ, с одной стороны, и полимерной матрицы с другой, можно предположить, что наблюдаемый эффект разупрочнения композитов связан с процессом последовательного разрыва волокон по мере роста приложенной нагрузки.

Наблюдаемый рост приложенной нагрузки на стадии деформационного упрочнения свидетельствует о том, что волокна в образце имеют разброс по уровню прочности, то есть с распределением прочностных характеристик волокна по длине. С ростом длины волокна в образце, а, следовательно, длины самого образца,

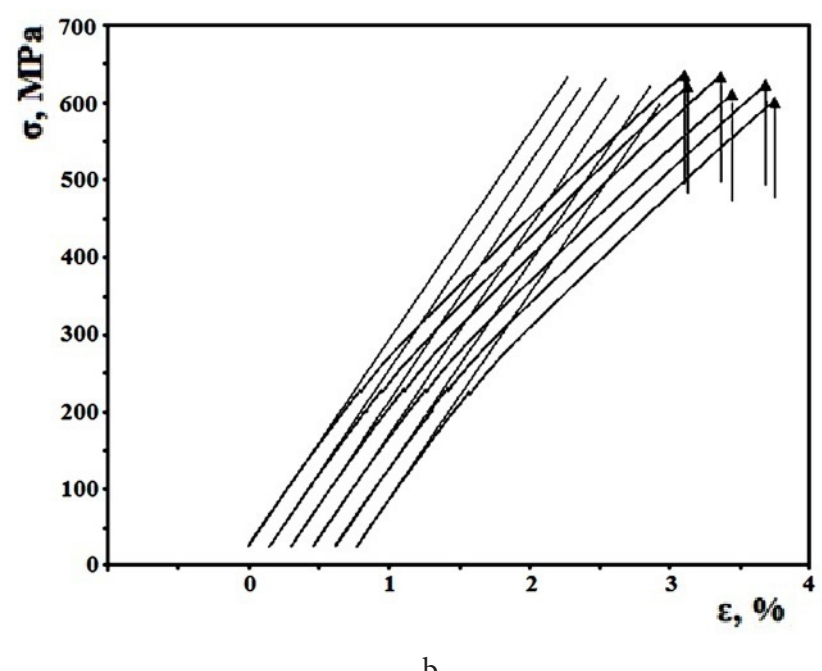

b

Рис. 1. Кривые $\sigma(\varepsilon)$ при растяжении для синтезированных композитов в матрице Т-107 с различными наполнителями: из углепластика Formosa TC-35-12K (a); стеклопластика Т-10-14 (b).

Fig. 1. The $\sigma(\varepsilon)$ curves under tension for the synthesized composites in the T-107 matrix with various fillers: from Formosa TC-35-12K carbon fiber (a); T-10-14 fiberglass (b). 
растет вероятность появления участков нити с все более низким уровнем прочности. При этом в общем случае должен понижаться измеряемый в эксперименте предел упругости и, как следствие, предел прочности композита. Эта зависимость прочностных характеристик композита от геометрических параметров изделия определяется функцией распределения прочности волокна по его длине. Для нахождения этой функции можно воспользоваться полученными экспериментально деформационными кривыми образцов композитов.

Кривую растяжения композита, углеродные или стеклянные волокна в котором расположены вдоль оси образца, можно схематично представить в виде, показанном на Рис. 3.

На протяжении всей деформационной кривой основной вклад в величину напряжения обеспечивают волокна и, в значительно меньшей степени, полимерная матрица, предел прочности которой в 21 раз меньше, чем волокон углерода, и в 5.5 раз чем стеклянных волокон. Кроме того, объемная доля волокон в композитах превышает объемную долю связующего полимера в 2 раза. В рамках изложенных представлений, напряжение растяжения композита $\sigma_{k}(\varepsilon)$ вплоть до его разрушения определяется суммарным напряжением растяжения наполнителя $\sigma$, (еще не разрушенных волокон в процессе растяжения), и величиной упругого напряжения $\sigma_{m}$ со стороны полимерной матрицы: $\sigma_{k}(\varepsilon)=\sigma(\varepsilon)+\sigma_{m}(\varepsilon)$. Результирующее напряжение растяжения рассчитывается с учетом доли площади поперечного сечения образца занятой матрицей и наполнителем. Зависимость напряжения растяжения композита $\sigma_{k}$ с учетом упругой деформации полимерной матрицы от величины деформации можно выразить в виде:

$$
\sigma_{k}(\varepsilon)=n(\sigma) S_{B} E_{B} \varepsilon+E_{m} \varepsilon .
$$

Здесь

$$
n(\sigma) S_{B} E_{B}=E^{\prime}(\sigma)
$$

- эффективный модуль упругости наполнителя композита; $n(\sigma)$ - число нитей пересекающих единицу площади поперечного сечения образца при достижении напряжения растяжения $\sigma ; S_{B}-$ площадь поперечного сечения волокна; $E_{B}-$ модуль упругости волокна; $\varepsilon-$ упругая деформация образца, равная по величине упругой деформации волокна $\varepsilon=\varepsilon_{B}$.

Эффективный модуль упругости полимерной матрицы

$$
E_{m}=\left(1-S_{B} n_{0}\right) E_{m}
$$

зависит от ее объемной доли в композите, и не зависит от деформации $\varepsilon ; n_{0}-$ исходное до деформации число волокон, пересекающих единицу площади образца; $E_{m}$ - модуль упругости материала полимерной матрицы. Предполагается, что после разрыва волокна примыкающая к нему полимерная область не изменяет свое напряженное состояние.

В области упругой деформации на участке ОА Рис. 3 число нитей на единицу площади сечения оставалось неизменным $n(\sigma)=n_{0}$. При деформациях, превышающих предел упругости $\varepsilon>\varepsilon$, происходит последовательный процесс разрушения волокон. С ростом деформации $\varepsilon$

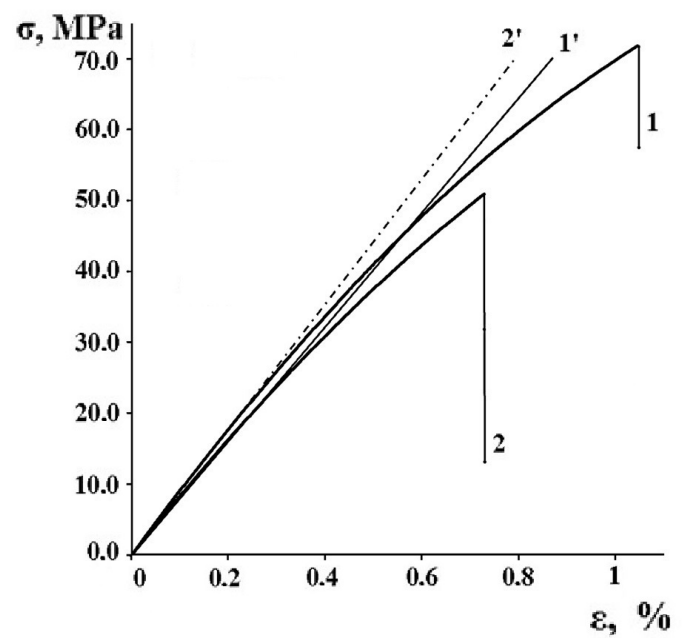

Рис. 2. Кривые $\sigma(\varepsilon)$ при растяжении для образцов 1 и 2 материала матрицы Т-107.

Fig. 2. Curves $\sigma(\varepsilon)$ under tension for samples 1 and 2 in the T-107 matrix.

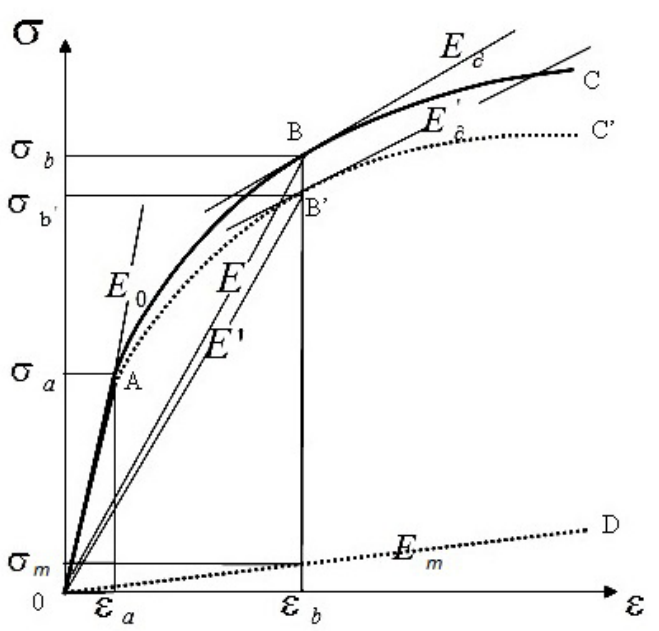

Рис. 3. Характерный вид деформационной кривой растяжения композита.

Fig. 3. Characteristic form composite tensile deformation curve.

$\left(\varepsilon>\varepsilon_{a}\right)$ уменьшается число $n$ несущих нагрузку волокон, однако средний уровень напряжения растяжения $\sigma$ продолжает расти за счет упругого растяжения оставшихся волокон.

Дифференцируя (3) по деформации $\varepsilon$, получим выражение для динамического модуля упругости $d \sigma_{k} / d \varepsilon=E_{\partial}$ в виде:

$$
\frac{d \sigma_{k}}{d \varepsilon}=E_{\partial}=E_{B} S_{B}\left(n+\frac{\partial n}{\partial \sigma} \frac{\partial \sigma}{\partial \varepsilon} \varepsilon\right)+E_{m},
$$

касательной в любой точке деформационной кривой, как это показано на Рис. 3. Величину динамического модуля $E_{\partial}$ композита можно определить по углу наклона. Динамический модуль, определяемый только волокнами без учета матрицы, равен

$$
E_{\partial}^{\prime}=E_{\partial}-\bar{E}_{m} .
$$

Величина $E^{\prime}=(\partial \sigma / \partial \varepsilon)_{n} \quad-\quad$ модуль упругости, определяемый только упругой деформацией остав- 
шихся после частичного разрушения волокон. С учетом вклада матрицы, величину модуля можно определить для каждой точки деформационной кривой по формуле:

$$
E^{\prime}=\frac{\sigma}{\varepsilon}-E_{m}=E-E_{m},
$$

где $E-$ модуль упругости композита при сохранении числа волокон (например, при разгрузке).

Производная $(\partial n / \partial \sigma)$ является функцией распределения волокон в образце по усредненной величине критического напряжения их разрушения. Величина эта отрицательная, так как с увеличением напряжения число не разрушенных волокон $n$ уменьшается. Изменив знак производной на положительный, то есть, интерпретируя $(\partial n / \partial \sigma)$ как рост числа оборванных волокон с ростом напряжения, из соотношения (5) с учетом (7), (8) получаем:

$$
\frac{\partial n}{\partial \sigma}=\frac{1}{S_{B} E_{B} \varepsilon}\left\{1-\frac{\left(E_{\partial}-E_{m}\right)}{\left(E-E_{m}\right)}\right\} .
$$

Исходя из соотношения (9) функцию распределения $g(f)$ вероятности обнаружения на единице длины волокна величину его прочности $f$ можно найти по формуле:

$$
g(f)=\frac{1}{L}\left(\frac{\partial n}{\partial \sigma}\right)=\frac{1}{f}\left\{1-\frac{\left(E_{\partial}-E_{m}\right)}{\left(E-E_{m}\right)}\right\},
$$

где $f=E_{B} S_{B} \varepsilon-$ сила натяжения волокна; $L-$ длина нити в образце.

Применяя экспериментальные данные для исследуемых композитов на Рис. 2 по формуле (10) можно оценить функцию распределения $g(f)$ вероятности обнаружения на единице длины волокна величину его прочности $f$.

Для оценки функции распределения $g(f)$ вероятности необходимо знать модуль упругости матрицы, который определим из кривой напряжение-деформация (Рис. 3).

C учетом полученного значения $E_{m}=3.8$ ГПа и принимая для углепластика значения $S_{B}=7 \cdot 10^{-6}$; $E_{B}=0.1$ ГПа; $\varepsilon=0.015 ; E_{\partial}=79$ ГПа; $E=160$ ГПа, получим $g(f)=0.05$.

Для стеклопластика, принимая значения $S_{B}=6 \cdot 10^{-6}$; $E_{B}=0.025$ ГПа; $\varepsilon=0.02 ; E_{\partial}=17$ ГПа; $E=27$ ГПа, получим $g(f)=0.14$.

Полученные значения имеют разумные величины. При этом вероятность обнаружения на единице длины волокна величины его прочности выше у стеклопластиков.

\section{4. Заключение}

Исследованы механические свойства стекло- и углепластиков с наполнителем Т-10-14 и Formosa TC-3512K, соответственно, в эпоксидной матрице Т-107 при испытании на растяжение. Установлено, что углепластики имеют более высокий условный предел прочности по сравнению со стеклопластиками. Предложена модель деформации полимерного композиционного материала, основанная на после- довательном разрыве волокон наполнителя по мере роста приложенной нагрузки. По полученным экспериментальным результатам сделана оценка функции распределения $g(f)$ вероятности обнаружения на единице длины волокна величины его прочности, величина которой выше у стеклопластиков.

Благодарность/Acknowledgements. Работа выполнена при поддержке Минобрнауки в рамках проектной части государственного задания (проект № 3.1867.2017/ПЧ). / This work has been supported by the Ministry of Education and Science as part of the project part of the state assignment (project No. 3.1867.2017/PCh)

\section{Литература/References}

1. E. N. Kablov. Aviation Materials and Technologies. 1 (34), 3 (2015). (in Russian) [Е.Н. Каблов. Авиационные материалы и технологии. 1 (34), 3 (2015).] Crossref

2. Y.-W. Mai, Zh.-Zh. Yu. In: Polymer nanocomposites. CRC Press, USA (2006). Crossref

3. A.F. Ab Ghani, J. Mahmud. Materials Science and Engineering Technology. 48 (3-4), 273 (2017). Crossref

4. B. Ostré, C. Bouvet, C. Minot, J. Aboissière. Composite Structures. 152, 768 (2016). Crossref

5. A.M. Kudrin, O.A. Karaeva, K.S. Gabriels, A. V. Solopchenko. VSTU Bulletin. 2 (14), 164 (2018). (in Russian) [А. М. Кудрин, О. А. Караева, К. С. Габриельс, А. В. Солопченко. Вестник ВГТУ. 2 (14), 164 (2018).]

6. C. Elanchezhian, B. Vijaya Ramnath, J. Hemalatha. Procedia Materials Science. 6, 1405 (2014). Crossref

7. O.I. Grishina, V.M. Serpova. Proceedings of VIAM. 1 (49), 41 (2017). (in Russian) [О.И. Гришина, В. М. Серпова. Труды ВИАМ. 1 (49), 41 (2017).]

8. M.L. Kerber, V.M. Vinogradov, G.S. Golovkin, A.A. Berlin. Polimernyye kompozitsionnyye materialy: struktura, svoystva, tekhnologiya (ed. by A. A. Berlin). Professiya, St-Petersburg (2011) 560 p. (in Russian) [М.Л. Кербер, В.М. Виноградов, Г.С. Головкин, А.А. Берлин. Полимерные композиционные материалы: структура, свойства, технология (под ред. А. А. Берлина). Профессия, СПб (2011) 560 с.]

9. S.L. Roginsky, M.Z. Kanovich, M.A. Koltunov. Vysokoprochnyye stekloplastiki. Khimiya, Moscow (1979) 144 p. (in Russian) [С.Л. Рогинский, М.3. Канович, M.А. Колтунов. Высокопрочные стеклопластики. Химия, Москва (1979) 144 с.]

10. Epoksidnyy prepreg T107. (in Russian) [Эпоксидный препрег T107]. http://www.inumit.ru/img/file/t107.pdf

11. O. V. Ovdak, Y.E. Kalinin, A.M. Kudrin, O.A. Karaeva, D. Y. Degtyarev. Inorganic Materials: Applied Research. 9 (1), 108 (2018). $\underline{\text { Crossref }}$

12. A.V. Kalgin, A.M. Kudrin, A.V. Solopchenko, M. Yu. Yablokova. VSTU Bulletin. 7 (11), 47 (2011). (in Russian) [А. В. Калгин, А. М. Кудрин, А. В. Солопченко, М. Ю. Яблокова. Вестник ВГТУ. 7 (11), 47 (2011).]

13. ASTM D 3039/D 3039M. Standard test method for tensile properties of polymer matrix composite materials.

14. ASTM D 638. Standard test method for tensile properties of plastics. 